\title{
Бимодальность в спектрах электролюминесценции InGaAs квантовых яма-точек
}

\author{
(C) А.А. Харченко ${ }^{1}$, А.М. Надточий ${ }^{1,2}$, А.А. Серин ${ }^{3}$, С.А. Минтаиров ${ }^{3}$, Н.А. Калюжный ${ }^{3}$, \\ A.E. Жуков ${ }^{2}$, М.B. Максимов ${ }^{1}$, S. Breuer ${ }^{4}$
}

${ }^{1}$ Санкт-Петербургский национальный исследовательский Академический университет им. Ж.И. Алфёрова

Российской академии наук,

194021 Санкт-Петербург, Россия

${ }^{2}$ Национальный исследовательский университет „Высшая школа экономики“, 190008 Санкт-Петербург, Россия

${ }^{3}$ Физико-технический институт им. А.Ф. Иоффре Российской академии наук,

194021 Санкт-Петербург, Россия

${ }^{4}$ Institute of Applied Physics, Technische Universität Darmstadt,

64289 Darmstadt, Germany

E-mail: antoshkerrr@gmail.com

Поступила в Редакцию 13 августа 2021 г.

В окончательной редакции 27 августа 2021 г.

Принята к публикации 27 августа 2021 г.

\begin{abstract}
Электролюминесценция волноводных структур на основе квантовых яма-точек была экспериментально исследована с поляризационным разрешением в диапазоне температур 60-300К. Установлено, что излучение из основного состояния состоит из двух пиков с разной степенью ТЕ-поляризации, максимумы которых сближаются с понижением температуры. Бимодальность в спектрах электролюминесценции мы связываем с существованием двух различных объектов в активной области: квантовых яма-точек, которые имеют частично ТЕ-поляризованное излучение, и квантовых точек, излучающих почти полностью ТЕ-поляризованный свет.
\end{abstract}

Ключевые слова: электролюминесценция, квантовые точки, InGaAs-гетероструктуры, волноводные структуры, поляризация излучения.

DOI: $10.21883 /$ FTP.2022.01.51818.9730

\section{1. Введение}

Гетероструктуры на основе InGaAs квантовых ям (КЯ) и InAs квантовых точек (КТ), выращиваемые на подложках GaAs, в настоящее время широко используются в различных областях науки и техники, в частности в полупроводниковых фотоэлектрических преобразователях и светоизлучающих устройствах [1]. Данная работа посвящена исследованию недавно разработанных структур переходной размерности, называющихся квантовыми яма-точками (КЯТ) [2]. КЯТ представляют собой InGaAs/GaAs квантовую яму с ярко выраженной модуляцией по составу индия и толщине. КЯТ можно также рассматривать как сверхплотный массив InGaAs КТ с малой энергией локализации. Сохраняя ключевые особенности КЯ и КТ, квантовые яма-точки обладают некоторыми специфическими преимуществами, например, имеют гораздо более высокое усиление (поглощение) по сравнению с квантовыми точками InAs/GaAs и даже квантовыми ямами [3]. Кроме того, имеется возможность последовательно выращивать более 15 слоев КЯТ без образования дислокаций, что в случае КЯ требует использования технологически сложных методов компенсации упругих напряжений. Вышеперечисленные особенности КЯТ позволили использовать их в $\mathrm{GaAs}$ фотоэлектрических преобразователях, показавших рекордный прирост фототока [4], а также микродисковых лазерах, продемонстрировавших рекордно высокую выходную мощность и быстродействие $[5,6]$. В силу относительной новизны и большой сложности адекватного компьютерного моделирования КЯТ еще мало изучены, что делает актуальным детальное исследование их свойств.

В данной работе исследуется электролюминесценция (ЭЛ) волноводных структур с КЯТ. В спектрах фотолюминесценции (ФЛ) с поверхности структур с аналогичными КЯТ ранее наблюдались спектральные особенности, связанные с бимодальностью их распределения по размеру [7]. Однако влияние бимодальности КЯТ на спектры ЭЛ волноводных структур, конструкция которых типична для таких широко используемых полупроводниковых приборов, как торцевые лазеры, усилители, волноводные детекторы и т.д., ранее не исследовалось. В данной работе исследована электролюминесценция с поляризационным разрешением волноводных структур с КЯТ и обнаружено два типа нанообъектов, имеющих разную поляризацию.

\section{2. Эксперимент}

Для проведения экспериментального исследования была синтезирована волноводная $p-i-n$-структура 


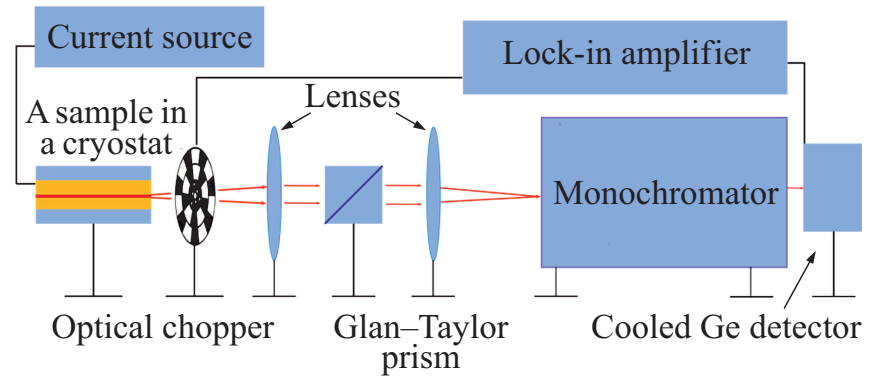

Рис. 1. Схема экспериментальной установки.

InGaAs/AlGaAs, выращенная на вицинальной подложке $\mathrm{GaAs}(100)$ методом газофазной эпитаксии из металлорганических соединений. Активная область представляла собой 10 слоев квантовых яма-точек, разделяемых 40-нанометровыми GaAs барьерами. Каждый из слоев КЯТ сформирован осаждением 8 монослоев $\mathrm{In}_{0.4} \mathrm{Ga}_{0.6} \mathrm{As}$. Из эпитаксиальной структуры были изготовлены образцы, представляющие собой полосковые волноводы длиной 1.5 мм и шириной 50 мкм, конструкция которых типична для полупроводниковых торцевых лазеров. С подробными деталями роста и постростовой обработки можно ознакомиться в работе [1]. Схема экспериментальной установки представлена на рис. 1. В гелиевый криостат замкнутого цикла был помещен образец, к которому подведены электрические контакты. Излучение с торца образца, последовательно проходя через поляризатор и спектральный прибор (МДР-23), регистрировалось охлаждаемым Ge-фотодиодом в режиме синхронного детектирования. Измерения проводились при низких (на порядок ниже величины порога лазерной генерации) токах накачки образца, чтобы уменьшить долю вынужденного излучения.

\section{3. Результаты и обсуждение}

В результате измерений были получены поляризационно-разрешенные спектры ЭЛ в температурном диапазоне 60-300 К. На рис. 2 приведены спектры для двух углов поворота поляризатора: $0^{\circ}-\mathrm{TE}$ (ось пропускания поляризатора параллельна плоскости волновода) и $90^{\circ}$ - ТМ, а также спектра, полученного без использования поляризатора при температуре $240 \mathrm{~K}$ и плотности тока инжекции $20 \mathrm{~A} / \mathrm{cm}^{2}$. В спектрах, полученных без поляризационного разрешения, достаточно тяжело определить наличие коротковолнового пика, который проявляется как слабовыраженное плечо (рис. 2). В то же время при детектировании излучения в ТЕ- и ТМ-поляризациях достаточно уверенно удается разрешить два пика с разными длинами волн. Для того чтобы наиболее точно разделить эти два пика, а также определить степени их поляризации, спектры были представлены в виде трехмерной поверхности, отображающей зависимость интенсивности излучения от угла поворота поляризатора $\theta$ и длины волны $\lambda$ (рис. 3,a). Данная поверхность была аппроксимирована следующим аналитическим выражением, представляющим собой сумму двух частично поляризованных гауссовых пиков:

$$
I(\theta, \lambda)=P G_{1}(\theta, \lambda)+P G_{2}(\theta, \lambda) .
$$

В (1) частично поляризованная функция Гаусса представлена следующим, основанным на законе Малюса, выражением:

$$
P G_{i}=\left(a_{i}+b_{i} \cos ^{2} \frac{\pi\left(\theta_{0 i}-\theta\right)}{180}\right) e^{-\frac{\left(\lambda-\lambda_{i}\right)^{2}}{2 w_{i}^{2}}},
$$

где параметрами аппроксимации являются $a_{i}, b_{i}$, положение $i$-го пика $\lambda_{i}$, его полуширина $w_{i}$ и угол $\theta_{0 i}$, показывающий направление линейной поляризации (0-TM, 90-TE). В таком случае степень поляризации $D o P_{i}$ соответствующего пика можно вычислить по формуле:

$$
D o P_{i}=\frac{I_{\max }+I_{\min }}{I_{\max }-I_{\min }}=\frac{2 a_{i}+b_{i}}{b_{i}} .
$$

На рис. 3, $b$ приведены сечения аппроксимирующей поверхности в $\mathrm{TE}\left(0^{\circ}\right)$-и ТМ $\left(90^{\circ}\right)$-поляризациях, а также соответствующие данные ЭЛ. Можно видеть, что более интенсивный длинноволновый пик аппроксимируется достаточно хорошо, в то время как аппроксимация менее интенсивного коротковолнового пика недостаточно качественная. Мы связываем это с отклонением реальной формы пиков ЭЛ от гауссовой, которая была использована в модели. Однако мы предполагаем, что полученные с помощью такой аппроксимации зависимости в достаточной мере отражают температурные тенденции реальных пиков в спектрах ЭЛ.

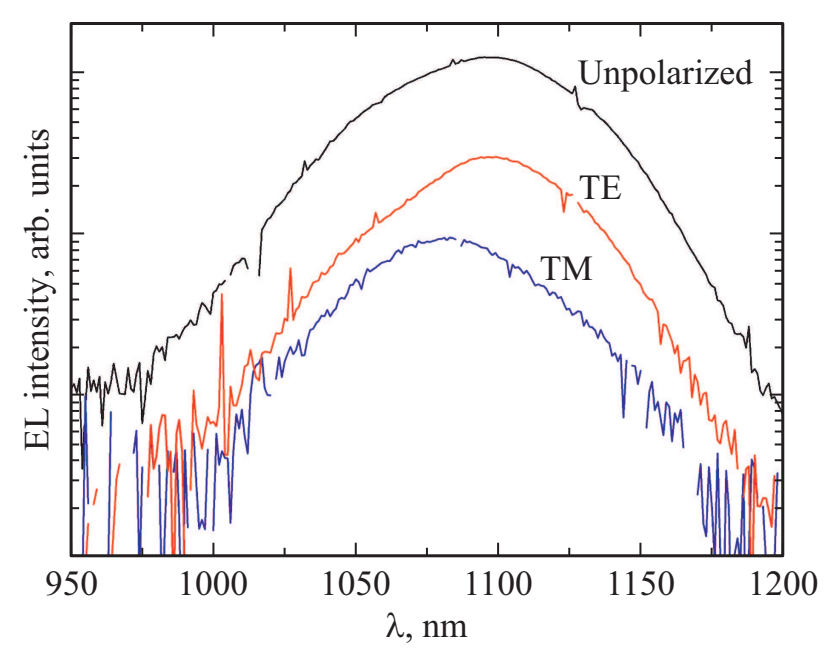

Рис. 2. Экспериментальные спектры ЭЛ при температуре $240 \mathrm{~K}$ для неполяризованного (черная кривая), ТЕ $\left(0^{\circ}\right)-$ и $\mathrm{TM}\left(90^{\circ}\right)$-поляризованного излучения (красная и синяя кривые соответственно). 

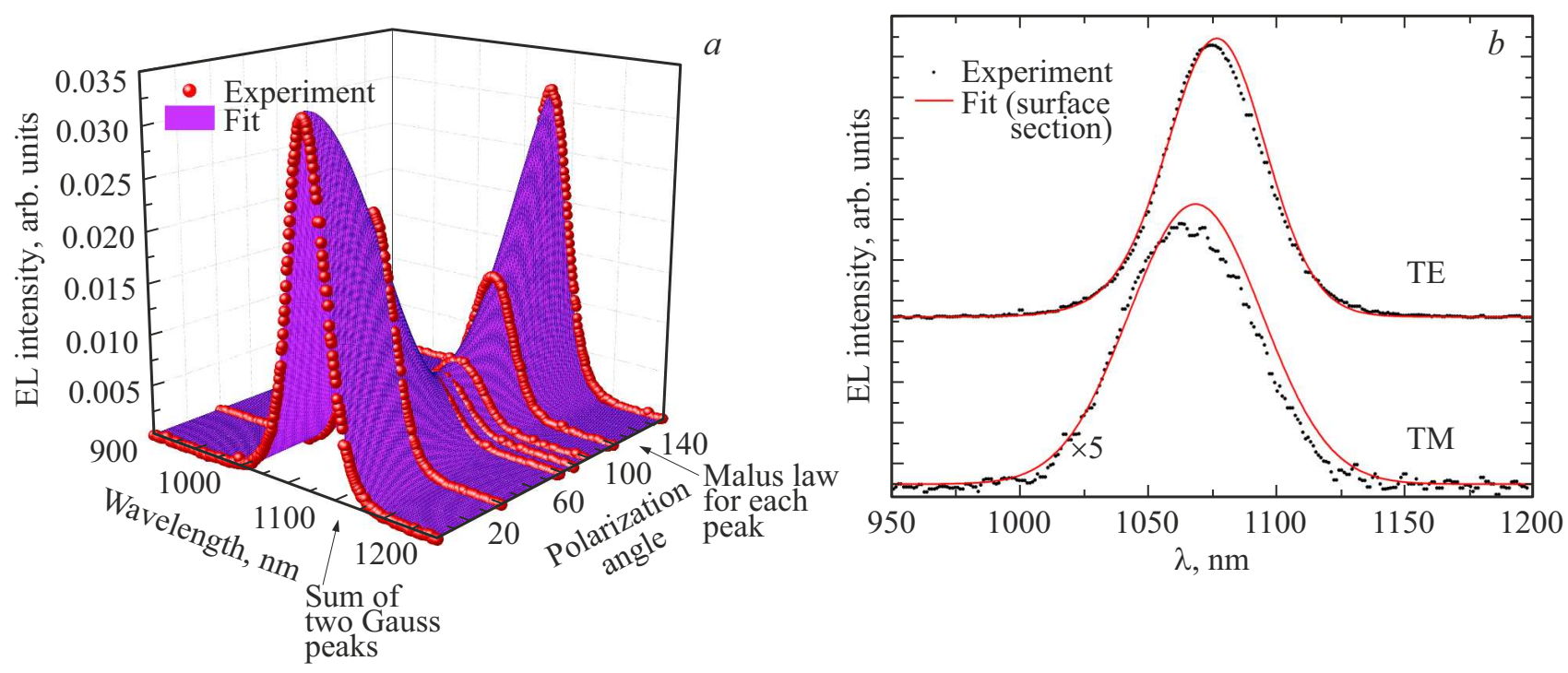

Рис. 3. Иллюстрация аппроксимации поверхностью экспериментальных данных при температуре $100 \mathrm{~K}(a)$. Сечения модельной поверхности и экспериментальные данные для ТЕ $\left(0^{\circ}\right)$ - и ТМ $\left(90^{\circ}\right)$-поляризаций $(b)$.

Температурные зависимости длины волны пиков в спектрах ЭЛ, полученные при помощи аппроксимаций, представлены на рис. 4. Из рис. 4, $a$ видно, что коротковолновый и длинноволновый пики сближаются с понижением температуры. Похожее поведение ранее наблюдалось нами при исследованиях образцов с КЯТ методом ФЛ [7], где было показано, что более коротковолновая линия ФЛ соответствует излучению из массива квантовых яма-точек, в то время как длинноволновая линия связана с изучением из объектов большего размера: квантовых точек, сходных по геометрии с классическими точками, формируемыми в режиме роста Странского-Крастанова. Наличие двух ансамблей наноструктур разной формы и размера подтвердилось изображениями, полученными методом просвечивающей электронной микроскопии. Таким образом, и в спектрах ЭЛ мы связываем длинноволновый пик с излучени-

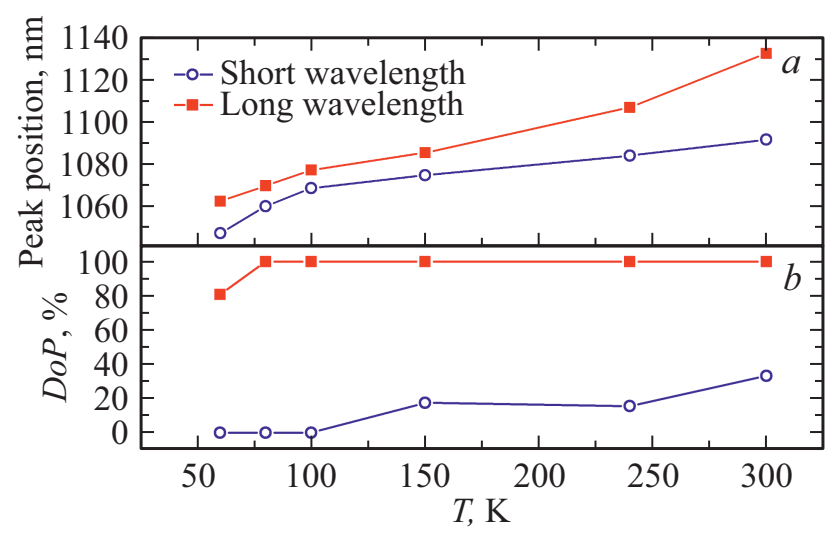

Pис. 4. Температурные зависимости длины волны пиков в спектре ЭЛ $(a)$ и степень их поляризации $(b)$, определенные в результате аппроксимации. ем квантовых яма-точек, а длинноволновый пик - с излучением квантовых точек. На рис. $4, b$ представлены температурные зависимости степени поляризации двух данных пиков: длинноволновый пик (КТ) практически полностью ТЕ-поляризован, в то время как у коротковолнового пика (КЯТ) наблюдается частичная ТЕ-поляризация при комнатной температуре, которая при понижении температуры пропадает.

Отметим, что обнаруженные в данной работе пики в излучении ЭЛ находятся в области основного состояния КЯТ, для которого предыдущие исследования планарных структур показали ярко выраженную ТЕ-селекцию в поглощении [8], в то время как переходы с легкой дыркой соответствуют значительно меньшим длинам волн [9]. Обнаружение в массиве КЯТ объектов, обладающих почти неполяризованным излучением, является достаточно необычным и может иметь высокую практическую значимость для реализации волноводных оптических усилителей и источников излучения, не обладающих поляризационной чувствительностью. Как правило, квантово-размерная активная среда, в первую очередь квантовая яма, но и среды другой размерности в системе материалов InGaAs имеют ярко выраженную ТЕ-поляризацию основного оптического перехода вследствие особенностей переходов с тяжелой дыркой. Чтобы реализовать взаимодействие таких сред с ТМ-составляющей излучения, требуются специальные усилия, включая, например, создание многослойных массивов с вертикальным связыванием электронных уровней [10-12], использование различных подходов по управлению упругими напряжениями [13] и др. [14]. Применение КЯТ в качестве поляризационнонечувствительной среды может значительно упростить технологию создания таких приборов. 


\section{4. Заключение}

В результате исследований волноводных структур на основе квантовых яма-точек методом поляризационноразрешенной электролюминесценции установлено, что излучение КЯТ в основном состоянии состоит из двух пиков разной степени линейной поляризации, которые мы связываем с наличием двух типов объектов в активной области - КЯТ и квантовых точек, излучающих в более длинноволновой области спектра. Излучение квантовых точек является полностью ТЕ-поляризованным, а излучение квантовых яма-точек практически не поляризованным, что может оказаться важным как для понимания фундаментальных свойств электронной структуры КЯТ, так и для реализации поляризационнонечувствительных оптоэлектронных приборов.

\section{Финансирование работы}

Работа поддержана Министерством науки и высшего образования РФ, проект 0791-2020-0002, а также грантом РФФИ 18-502-12081 ННИО_а. Анализ данных проводился в рамках Программы фундаментальных исследований НИУ ВШЭ. Автор S.B. благодарит за поддержку German Research Foundation (DFG) (project № 389193326).

\section{Конфликт интересов}

Авторы заявляют, что у них нет конфликта интересов.

\section{Список литературы}

[1] M.V. Maximov, A.M. Nadtochiy, S.A. Mintairov, N.A. Kalyuzhnyy, N.V. Kryzhanovskaya, E.I. Moiseev, N.Y. Gordeev, Y.M. Shernyakov, A.S. Payusov, F.I. Zubov, V.N. Nevedomskiy, S.S. Rouvimov, A.E. Zhukov. Appl. Sci., 10, 1038 (2020).

[2] S.A. Mintairov, N.A. Kalyuzhnyy, M.V. Maximov, A.M. Nadtochiy, S.S. Rouvimov, A.E. Zhukov. Electron. Lett., 511602 (2015).

[3] N.Y. Gordeev, M.V. Maximov, A.S. Payusov, A.A. Serin, Y.M. Shernyakov, S.A. Mintairov, N.A. Kalyuzhnyy, A.M. Nadtochiy, A.E. Zhukov. Semicond. Sci. Technol., 36, 015008 (2021).

[4] S.A. Mintairov, N.A. Kalyuzhnyy, A.M. Nadtochiy, M.V. Maximov, V.N. Nevedomskiy, L.A. Sokura, S.S. Rouvimov, M.Z. Shvarts, A.E. Zhukov. Semiconductors, 52, 1249 (2018).

[5] E.I. Moiseev, N.V. Kryzhanovskaya, M.V. Maximov, F.I. Zubov, A.M. Nadtochiy, M.M. Kulagina, Y.M. Zadiranov, N.A. Kalyuzhnyy, S.A. Mintairov, A.E. Zhukov. Optics Lett., 43, 4554 (2018).

[6] F.I. Zubov, M.V. Maximov, N.V. Kryzhanovskaya, E.I. Moiseev, M.E. Muretova, A.A. Mozharov, N.A. Kalyuzhnyy, S.A. Mintairov, M.M. Kulagina, N.N. Ledentsov, L. Chorchos, N.N. Ledentsov, A.E. Zhukov. Optics Lett., 44, 5442 (2019).

[7] А.М. Надточий, С.А. Минтаиров, Н.А. Калюжный, С.С. Рувимов, Ю.М. Шерняков. ФТП, 49, 1115 (2015).
[8] A.A. Kharchenko, A.M. Nadtochiy, S.A. Mintairov, Y.M. Shernyakov, A.A. Serin, N.Y. Gordeev, M.V. Maximov, A.E. Zhukov. Nano-Structures and Nano-Objects, 25, 100628 (2021).

[9] S.A. Mintairov, N.A. Kalyuzhnyi, M.V. Maksimov, A.M. Nadtochiy, A.A. Kharchenko, M.Z. Shvarts, A.E. Zhukov. Techn. Phys. Lett., 46, 203 (2020).

[10] T. Kita, M. Suwa, T. Kaizu, Y. Harada. J. Appl. Phys., 115 (23), 233512 (2014).

[11] P. Yu, W. Langbein, K. Leosson, J.M. Hvam, N.N. Ledentsov, D. Bimberg, V.M. Ustinov, A.Yu. Egorov, A.E. Zhukov, A.F. Tsatsul'nikov, Yu.G. Musikhin. Phys. Rev. B, 60 (24), 680 (1999).

[12] Li.L. Ridha, M. Rossetti, G. Patriarche, A. Fiore. Optical Quant. Electron., 40 (2-4), 239 (2008).

[13] W.C.H. Choy. IEEE J. Quant. Electron., 36, 164 (2000).

[14] P. Jayavel, H. Tanaka, T. Kita, O. Wada, H. Ebe, M. Sugawara, J. Tatebayashi, Y. Arakawa, Y. Nakata, T. Akiyama. Appl. Phys. Lett., 84, 1820 (2004).

Редактор А.Н. Смирнов

\section{Bimodality in the electroluminescence spectra of quantum well-dots InGaAs nanostructures}

A.A. Kharchenko ${ }^{1}$, A.M. Nadtochiy 1,2, A.A. Serin ${ }^{3}$ S.A. Mintairov ${ }^{3}$, N.A. Kalyuzhnyy ${ }^{3}$, A.E. Zhukov ${ }^{2}$, M.V. Maximov' ${ }^{1}$, S. Breuer ${ }^{4}$

${ }^{1}$ Alferov University, 194021 St. Petersburg, Russia

${ }^{2}$ National Research University Higher School of Economics, 190008 St. Petersburg, Russia ${ }^{3}$ loffe Institute, 194021 St. Petersburg, Russia

${ }^{4}$ Institute of Applied Physics, Technische Universität Darmstadt, 64289 Darmstadt, Germany

Abstract The electroluminescence spectra of waveguiding structures based on quantum well-dots were investigated with polarization resolution in the temperature range of $60-300 \mathrm{~K}$. It is found that the ground state emission consists of two peaks with different degrees of TE-polarization and these peaks are getting closer with temperature decrease. We attribute the bimodality to the existence of two different types of nanoobjects in the active region: the quantum well-dots, which have partially TE-polarized emission, and quantum dots emitting almost fully TE-polarized light. 